\section{Identification of the atypically modified autoantigen Ars2 as the target of B-cell receptors from activated B-cell-type diffuse large B-cell lymphoma}

\author{
Lorenz Thurner, ${ }^{1}$ Sylvia Hartmann, ${ }^{2}$ Moritz Bewarder, ${ }^{1}$ Natalie Fadle, ${ }^{1}$ Evi \\ Regitz, ${ }^{1}$ Claudia Schormann, ${ }^{1}$ Natalia Quiroga,${ }^{1}$ Maria Kemele, ${ }^{1}$ Wolfram \\ Klapper, ${ }^{3}$ Andreas Rosenwald, ${ }^{4}$ Lorenz Trümper, ${ }^{5}$ Rainer Maria Bohle, ${ }^{6}$ Anna \\ Nimmesgern, ${ }^{7}$ Christina Körbel, ${ }^{8}$ Matthias W. Laschke, ${ }^{8}$ Michael D. Menger, ${ }^{8}$ \\ Stefan Barth, ${ }^{9}$ Boris Kubuschok, ${ }^{10}$ Anja Mottok, ${ }^{11}$ Dominic Kaddu-Mulindwa, ${ }^{1}$ \\ Martin-Leo Hansmann, ${ }^{2}$ Viola Pöschel, ${ }^{1}$ Gerhard Held, ${ }^{12}$ Niels Murawski, ${ }^{1}$ \\ Stephan Stilgenbauer, ${ }^{1}$ Frank Neumann, ${ }^{1}$ Klaus-Dieter Preuss ${ }^{1}$ and Michael \\ Pfreundschuh $^{1+}$
}

${ }^{1}$ Saarland University Medical School, José Carreras Center for Immuno- and Gene Therapy and Internal Medicine I, Homburg/Saar, Germany; ${ }^{2}$ Dr. Senckenberg Institute of Pathology, Goethe University Hospital of Frankfurt am Main, Frankfurt am Main, Germany; ${ }^{3}$ Institute of Pathology, University of Kiel, Kiel, Germany; ${ }^{4}$ Institute of Pathology, University of Würzburg and CCC Mainfranken, Würzburg, Germany; ${ }^{5}$ Department of Hematology and Medical Oncology, University Hospital Göttingen, Göttingen, Germany; 'Saarland University Medical School, Institute of Pathology, Homburg/Saar, Germany; ${ }^{7}$ Institute of Medical Microbiology and Hygiene, University of Saarland, Homburg, Germany; ${ }^{8}$ Institute for Clinical \& Experimental Surgery, University of Saarland, Homburg/Saar, Germany; 'Institute for Infectious disease \& Molecular Medicine, University of Cape Town, Cape Town, South Africa; ${ }^{10}$ Department of Internal Medicine II, Augsburg University Medical Center, Augsburg, Germany; ${ }^{11}$ Institute of Human Genetics, UIm University and UIm University Medical Center, UIm, Germany and ${ }^{12}$ Department of Hematology/Oncology, Westpfalzklinikum, Kaiserslautern, Germany

${ }^{\dagger}$ Deceased.

\section{ABSTRACT}

I has been suggested that stimulation of B-cell receptors (BCR) by specific antigens plays a pathogenic role in diffuse large B-cell lymphoma (DLBCL). Here, it was the aim to screen for specific reactivities of DLBCL-BCR in the spectrum of autoantigens and antigens of infectious origin. Arsenite resistance protein 2 (Ars2) was identified as the BCR target of three of five activated B-cell type DLBCL cell lines and two of 11 primary DLBCL cases. Compared to controls, Ars2 was hypophosphorylated exclusively in cases and cell lines with Ars2-specific BCR. In a validation cohort, hypophosphorylated Ars2 was found in eight of 31 activated B-cell type DLBCL, but in only one of 20 germinal center B-cell like type DLBCL. Incubation with Ars2 induced BCR-pathway activation and increased proliferation, while an Ars2/ETA' toxin conjugate induced killing of cell lines with Ars2-reactive BCR. Ars2 appears to play a role in a subgroup of activated B-cell-type DLBCL. Moreover, transformed DLBCL lines with Ars2-reactive BCR still showed growth advantage after incubation with Ars2. These results provide knowledge about the pathogenic role of a specific antigen stimulating the BCR pathway in DLCBL.

\section{Introduction}

Diffuse large B-cell lymphoma (DLBCL) is the most common aggressive B-cell nonHodgkin lymphoma. According to the World Health Organization (WHO) classification, DLBCL can be classified based on gene expression profiling (GEP) into an activated $\mathrm{B}$-cell $(\mathrm{ABC})$-like type, a germinal center B-cell (GCB)-like type and primary mediastinal B-cell lymphoma. ${ }^{1,2}$ In contrast to relatively well-studied genetic or epigenetic pathway alterations, little is known about specific and complementary external 
stimuli of different subgroups of DLBCL., In particular, DLBCL of the ABC-type or the recently specified MCDtype or Cluster 5 harbor recurrent mutations in MYD 88 and $C D 79 B$ genes with dependency on constitutive BCR signaling.5.8 For primary central nervous system lymphoma, which represents a specific extranodal subtype of DLCBL with molecular similarities to MCD type or C5 with frequent mutations in MYD88 and CD79,9,10 SAMD14/neurabin-I were recently identified as antigens of $\mathrm{BCR}$, and were hyper-N-glycosylated specifically in patients with SAMD14/neurabin-I-reactive BCR. ${ }^{11}$ In systemic DLBCL, a cis and trans stimulation of the BCR by a so far uncharacterized autoantigen was reported for the HBL1 line. Moreover, an anti-idiotype reactivity of complementarity determining region 3 of the BCR of the TMD8 line against an epitope within its own FR2 $\left(\mathrm{V}^{37} \mathrm{R}^{38}\right)$ was described, and for U2932 and OCI-LY10 lines BCR reactivity against apoptotic cell debris was reported. ${ }^{8}$ This prompted us to screen for and characterize possible target antigens of BCR of systemic DLBCL using expression cloning of primary cryopreserved specimens and DLBCL lines and subsequent protein array screening. $12-14$

\section{Methods}

The study was approved by the local ethics committee (Ärztekammer des Saarlandes 12/13). For expression cloning of DLBCL-BCR, patients' snap-frozen specimens were obtained from the Dr. Senckenberg Institute of Pathology (Frankfurt am Main, Germany). Sera of a second cohort of patients with DLBCL were obtained from the DSHNHL RICOVER-60 trial.

DLBCL cryospecimens, of a third cohort of patients, of which the cell-of-origin had been determined by GEP, were obtained from the Institutes of Pathology of Würzburg and Kiel Universities.

\section{B-cell receptor screening for autoantigens}

BCR from nine DLBCL cell lines were prepared by digestion with papain. Moreover, expression cloning of recombinant BCR derived from primary DLBCL cryospecimens was performed, as described in the Online Supplementary Material. These DLBCL linederived BCR and the pooled recombinantly expressed BCR (each at a concentration of $10 \mu \mathrm{g} / \mathrm{mL}$ ) were screened on protein macroarrays containing clones of UniPEx 1 and 2 cDNA expression libraries (Bioscience, Dublin, Ireland), as previously described. ${ }^{13,14}$ To search for further antigens, all recombinant DLBCL-derived antigen-binding fragments (Fab) without reactivity against arsenite resistance protein 2 (Ars2) were screened against variously post-translationally modified UniPEx 1 and 2 protein macroarrays, including sumoylation, ubiquitination, citrullination, and acetylation. Protein macroarrays were sumoylated as described elsewhere ${ }^{15}$ and ubiquitination was performed with synchronized HeLa cell extracts. ${ }^{16}$ The screening for antigens of infectious origin is described in the Online Supplementary Material.

\section{Expression of target antigens and immunotoxins}

The expression clone of Ars2 and subsequently the epitopecontaining region consisting of amino acids 342-375 of Ars 2 were recombinantly expressed with a C-terminal FLAG tag by a pSFI vector in HEK293 cells. Additionally, C-terminally FLAG-tagged full-length Ars2 was transfected by electroporation into U2932 and TMD8 via a pRTS vector. ${ }^{17}$ C-terminally FLAG-tagged FamH83 and JmjD4 were recombinantly expressed in HEK293 cells. Site-directed mutagenesis of Ars2 and secondary modifica- tion of antigens is described in the Online Supplementary Material.

Immunotoxins with monomethyl auristatin $\mathrm{E}$ (MMAE) are effective in vivo and established in the clinics, but the synthesis of toxin conjugates with MMAE requires enzyme-cleavable dipeptide linkers and is therefore challenging for academic laboratories. ${ }^{18,19}$ Hence, a truncated form of Pseudomonas aeuroginosa exotoxin A (ETA') was used, as the ETA' conjugate can be recombinantly expressed directly. Recombinantly expressed immunotoxins, consisting of Ars2 amino acids 342-375 conjugated to ETA' were either obtained from the Fraunhofer Institute of Experimental Medicine and Immunotherapy (Aachen, Germany) or recombinantly expressed in our laboratory in E. coli BL21 and purified by the His-Tag, as described by Nachreiner et al. ${ }^{20}$

\section{Enzyme-linked immunosorbent assay (ELISA) for B-cell receptor and serum reactivity against target antigens and competition ELISA with apoptotic debris}

Ars2, ubiquitinated FamH83, and sumoylated JmjD4 were confirmed as BCR antigens by ELISA, as previously described. ${ }^{13}$ ELISA and competition assays with apoptotic debris are described in detail in the Online Supplementary Material.

\section{Western blot and isoelectric focusing}

Lysates of DLBCL lines or of whole blood from patients were loaded and separated by $10 \%$ sodium dodecylsulfate polyacrylamide gel electrophoresis and transferred to a polyvinylidene fluoride membrane using a transblot semidry transfer cell (Bio Rad). After blocking overnight at $4^{\circ} \mathrm{C}$ in phosphate-buffered saline $/ 10 \%$ nonfat dry milk, a recombinant Ars2-reactive His-tagged Fab was incubated at a concentration of $2 \mu \mathrm{g} / \mathrm{mL}$ for $1 \mathrm{~h}$ at room temperature, followed by incubation for $1 \mathrm{~h}$ at room temperature with murine anti-his antibody at a ratio of 1:2,000 (Qiagen), with horseradish peroxidase-labeled anti-mouse IgG antibody (Bio Rad). Chemiluminescence reagent (New England BioLabs) was used for immunoblot detection. Isoelectric focusing was performed as previously described. Proliferation, BCR pathway activation assays, cytotoxicity and apoptosis assays are described in the Online Supplementary Material.

\section{Results}

Recombinant BCR in the form of Fab were successfully synthesized from 11 DLBCL cases. Moreover, Fab of "natural" BCR were obtained by papain digestion from nine DLBCL cell lines. From three of these cell lines, recombinant Fab were generated (Online Supplementary Table S1).

\section{Screening of protein macroarrays and a library of infective agents}

The screening of DLBCL Fab identified an expression clone of Ars2 transcript variant 2 (RZPDp828K0526 from Unipex 2, UnigeneID: Hs.111801) spanning from amino acids 253 to 416 as the candidate antigenic target. The screening of the Fab of DLBCL cases and cell lines on posttranslationally modified protein macroarrays revealed sumoylated JmJD4 (RZPDp9027E0216D from Unipex 1; UnigeneID: Hs.555974) and ubiquitinylated FamH83 (RZPDp828G0328 from Unipex 2; UnigeneID: Hs.676336) as candidate antigens. The screening against bacterial lysates of 11 bacterial strains did not reveal any specific reactivity. Screening of an Infectious Disease Epitope Microarray (PEPperCHIP ${ }^{\circledast}$ Heidelberg, Germany) consisting of 3,760 database-derived B-cell epitopes associated with 196 pathogens, including various bacterial, fungal, parasitic, 
and viral pathogens, revealed no significant binding of the pooled DLBCL-BCR.

Confirmation of Ars2, sumoylated JmjD4, and ubiquitinated FamH3 as targets of diffuse large B-cell lymphoma B-cell receptors and determination of the B-cell receptor-binding epitope

ELISA with recombinant Ars2 (UnigeneID: Hs.111801) expressed with a C-terminal FLAG-tag in HEK293 confirmed Ars2 as the BCR target antigen from three of five (60\%) ABC-derived cell lines (OCI-Ly3, OCI-Ly10, and
U2932; but not HBL1 and TMD8) and none of four GCBDLBCL cell lines (Figure 1A). Recombinant BCR from two of 11 DLBCL cases (with unknown cell of origin), but none of nine mantle cell lymphomas and none of 11 primary central nervous system lymphomas were reactive with Ars2 (Figure 1B). Of the two Ars2-reactive DLBCL, one was a non-GCB type and one was unclassified according to immunohistochemistry using the Hans classifier. ${ }^{21}$ ELISA with fragments of different lengths of Ars2 as the coat identified a region spanning amino acids 350 to 416 as the BCR-binding epitope (Figure 1C), and all

A

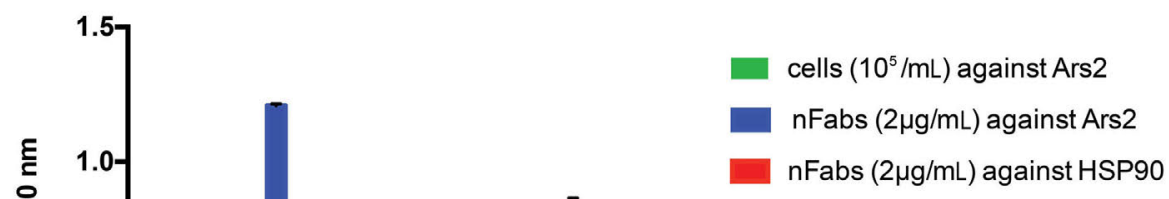

B

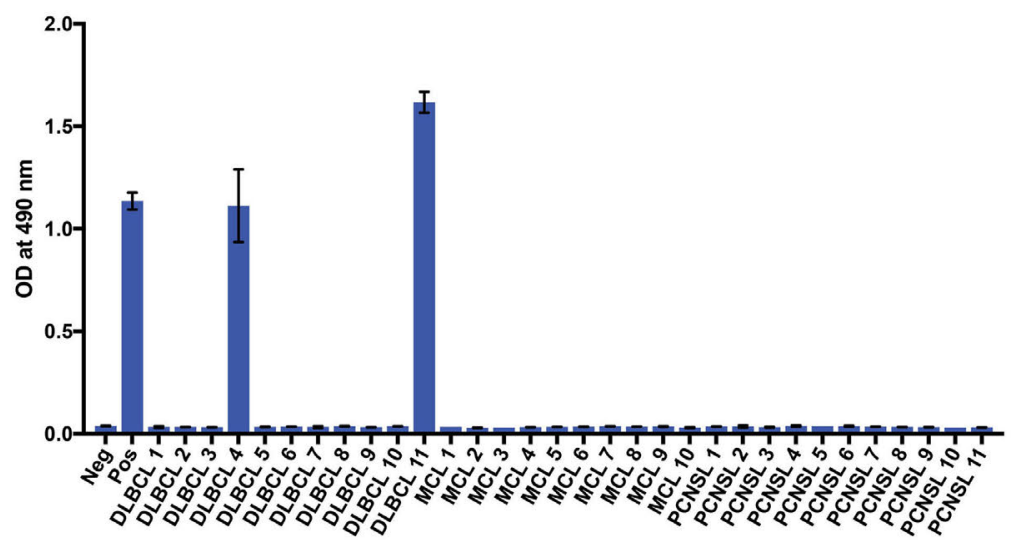

Figure 1. Reactivity of diffuse large $B$ cell lymphoma-derived B-cell receptors against Ars2. (A) Enzyme-linked immunosorbent assay (ELISA) for reactivity against Ars2 or sumoylated HSP9O as control of diffuse large B-cell lymphoma (DLBCL) cell line-derived natural (Papain-digested) B-cell receptors (BCR) in the Fab format and directly for cell membrane-bound BCR. The columns represent adsorbance at an optical density (OD) of $490 \mathrm{~nm}$ (mean and standard deviation). (B) ELISA for Ars2 reactivity of recombinant $B C R$ derived from primary DLBCL cryospecimens and controls. The columns represent adsorbance at OD $490 \mathrm{~nm}$ (mean and standard deviation). (C) Determination of the affinity region of BCR against Ars2: The affinity region within Ars2 was amino acids (AA) 301 416 with the highest observed affinity shown for AA 350-416. The columns represent adsorbance at OD $490 \mathrm{~nm}$, (mean and standard deviation). ABC: activated B-cell type; GC: germinal center B-cell type. (Figure continued on the next pages) 
D
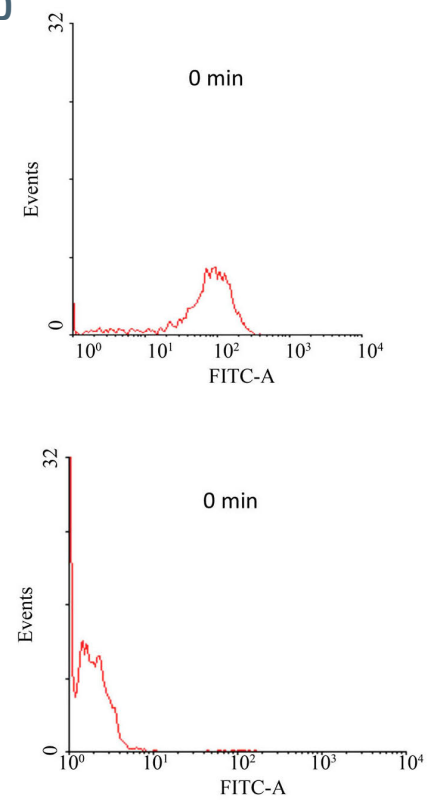

E

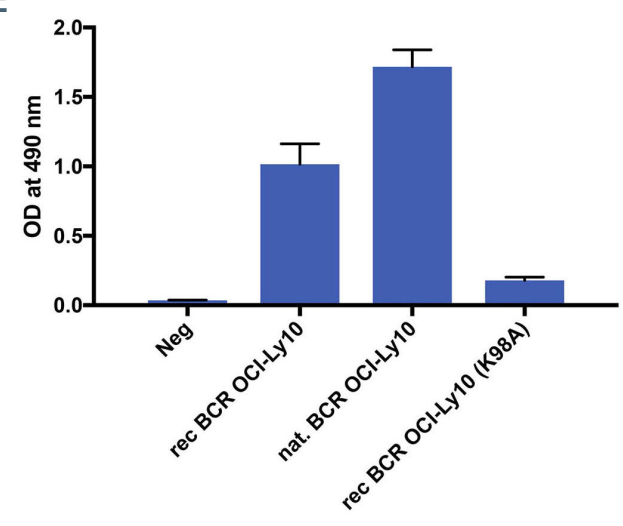

intracellular

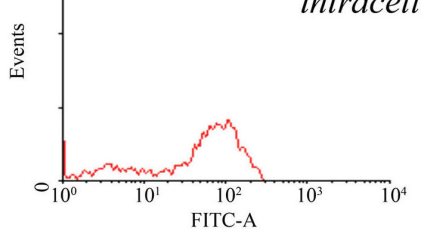

Figure 1. Continued from previous page. (D) Binding of Ars 2 and internalization into U2932 cells. At time 0 (left) Ars 2 (a fragment of 253 to 416 amino acids) bound to the cell surface of $\mathrm{U} 2932$ cells (above) and was not detected intracellularly (below). After 60 min incubation at $37^{\circ} \mathrm{C}$ (right) and washing, Ars 2 was no longer detected on the cell surface (above), but was found intracellularly (below). Non-specific binding was determined by incubation with antibodies alone. (E) ELISA for reactivity against Ars2 of the BCR of OCl-Ly10 wt and K98A. Recombinant and papain-digested (natural) Fab of OCl-Ly10 bound to Ars2. K98A mutagenesis of recombinant OCI-Ly10 BCR resulted in the loss of reactivity of the recombinant BCR against Ars2. K98A had previously been described for OCI-Ly10 as being responsible for loss of BCR autoreactivity against apoptotic debris. The columns represent adsorbance at OD $490 \mathrm{~nm}$ (mean and standard deviation).

Ars2-specific BCR derived from cell lines and cryospecimens bound to this epitope. ELISA with recombinant sumoylated JmjD4 or ubiquitinylated FamH83 expressed with a C-terminal FLAG-tag in HEK293 confirmed each as target antigens of one DLBCL-derived recombinant Fab and ubiquitinated FamH83 in addition as a target of a recombinant mantle-cell lymphoma-derived Fab (Online Supplementary Figures $S 1$ and S2). Fab did not bind to nonmodified JmjD4 and FamH83. Binding of Ars2 to membrane BCR was demonstrated by flow cytometry of DLBCL cell lines with Ars2-reactive BCR, but not for the TMD8 line without Ars2-reactive BCR. Internalization of C-terminally FLAG-tagged Ars2 into U2932 cells was observed after $60 \mathrm{~min}$ (Figure 1D). For OCI-Ly10, mutagenesis of K98A of the BCR heavy chain gene had been reported to result in loss of autoreactivity. ${ }^{8}$ OCI-Ly10 K98A resulted in loss of affinity against Ars2 (Figure 1E).

\section{Characterization of Ars2 in diffuse large B-cell lymphoma with Ars2-specific B-cell receptors}

No obvious differences in molecular weight of Ars2 from DLBCL cases with Ars2-reactive and Ars2-non-reactive BCR and controls were observed in western blots; similarly, Sanger sequencing revealed identical DNA sequences, excluding mutations in the coding sequence as an explanation for the immunogenicity of Ars2. However, isoelectric focusing of cell lines (Figure 2A) and DLBCL cases (Figure 2B) with an Ars2-reactive BCR revealed a less negatively charged Ars2 isoform. Dephosphorylation with alkaline phosphatase treatment led to a stronger reduction of the negative charges of Ars2 from DBLCL cases and cell lines without Ars2-specific BCR than in cases with Ars2-reactive BCR and resulted in the disappearance of the differences in electric charge between both isoforms of Ars2 (Figure 2C), demonstrating that the different isoelectric focusing pattern of Ars2 was due to hypophosphorylation in cases with Ars2-specific BCR. The hypophosphorylated Ars2 isoform was detected in all of the three DLBCL cell lines with Ars2reactive BCR (i.e., OCI-Ly3, OCI-Ly10, and U2932), but in none of six DLBCL lines without Ars2-reactive BCR. This association in the nine analyzed DLBCL cell lines between BCR reactivity against Ars2 and the presence of the hypophosphorylated isoform of Ars2 was statistically significant (Fisher exact $t$-test: two-tailed $P=0.0119$ ). Regarding the 11 DLBCL cases with recombinantly expressed Fab (Online Supplementary Table S1) derived from cryospecimens, only the two cases with Ars2-reactive Fab (\#3 and \#11) showed hypophosphorylation of Ars2. In this cohort of 11 cases of cryospecimens and recombinantly expressed $\mathrm{Fab}$, the association between Ars2 reactivity and presence of hypophosphorylated Ars2 isoform was also statistically significant (Fisher exact $t$ test: two-tailed $P=0.0182)$. Looking at the association between BCR reactivity of DLBCL cells and the presence of hypophosphorylated Ars2 isoform overall, considering data from both cell lines and cases with cryospecimenderived recombinant Fab, the association was statistically highly significant (Fisher exact $t$-test: two-tailed $P<0.0001$ ).

Hypophosphorylated Ars2 was detected in the biopsies of eight of $31(26 \%)$ ABC-type DLBCL cases characterized by GEP, but in only one of $20(5 \%)$ GCB-type DLBCL and in the peripheral blood from one in 100 healthy controls.

The hypophosphorylated sites were identified as serine 328 and serine 341 by site-directed point mutagenesis of various predicted sites in Ars2 transfected with a C-termi- 
nal FLAG tag in OCI-Ly10, OCI-Ly3 and, as a control, HBL1 (Figure 2D and Online Supplementary Figure S4).

\section{Frequency, titers, and IgG subclasses of Ars2 serum antibodies}

Ars2 antibodies were detected by ELISA in the sera of four of 98 patients with DLBCL, with titers ranging from 1:800 to $1: 1600$, and in one of 400 healthy controls. All four patients with Ars2 antibodies in their sera (\#22, \#27, \#41, \#73) were carriers of hypophosphorylated Ars2 in the cells of their peripheral blood (Figure 2B), but this isoform was not detected in the peripheral blood of any of the 94 other patients, resulting in a statistically significant relationship between serum Ars2-autoantibodies and the presence of the hypophosphorylated Ars2 in peripheral blood (Fisher exact $t$-test: two-tailed $P<0.0001$ ).

\section{Effects of Ars2 on diffuse large B-cell lymphoma lines}

Western blot analysis of BCR pathway activation after addition of recombinant Ars2 revealed a strong activation in the U2932 cell line with Ars2-reactive BCR, demonstrated by a strong upregulation of pTyr525/526 SYK, pTyr96 BLNK, pTyr759 PLC 2 , and pTyr223 BTK. Moreover, this BCR stimulation by Ars2 led to increases in MYC expression. However, regarding MYC two cases with Ars2-reactive BCR (\#4 and \#10) did not have MYCoverexpression, as determined by immunohistochemical analysis (data not shown). No BCR pathway activation was induced by the control antigen MAZ in the U2932 line or by addition of Ars2 to the HBL1 line (Figure 3A). Addition of recombinant Ars2 induced proliferation of U2932 and OCI-Ly3 cells, but not of DLBCL cell lines without Ars2reactive $\mathrm{BCR}$, such as TMD8, analyzed by the tetrazolium/formazan EZ4U assay (Figure 3B). This Ars2-induced growth stimulus could be reverted by addition of Ars2neutralizing recombinant $\mathrm{Fab}$ derived from patient \#4 (Figure 3C). Furthermore, flow cytometry analysis of U2932 cells showed a strong increase of cytoplasmic calcium levels after incubation with the Ars2 epitope, but not the control antigen (Figure 3D).

\section{Cytotoxicity of the Ars2/ETA' conjugate}

Addition of Ars2-ETA' resulted in inhibition of growth analyzed in proliferation assays. This inhibition could be reverted by preincubation of Ars $2 / \mathrm{ETA}^{\prime}$ toxin with the Ars2-reactive recombinant Fab derived from case \#4 (Figure 4A). The Ars2-ETA' conjugate exerted a specific and dose-dependent toxicity against the Ars2-reactive
A

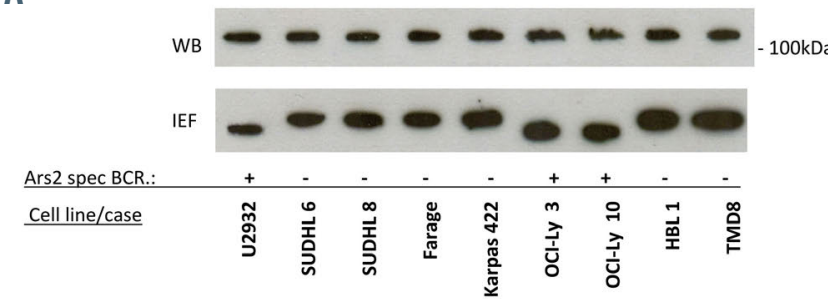

B
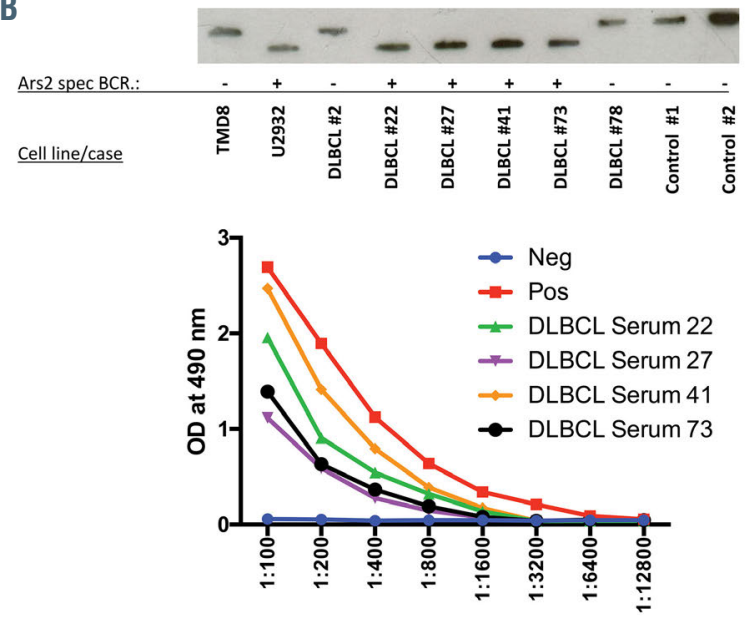

Titer of serum
C

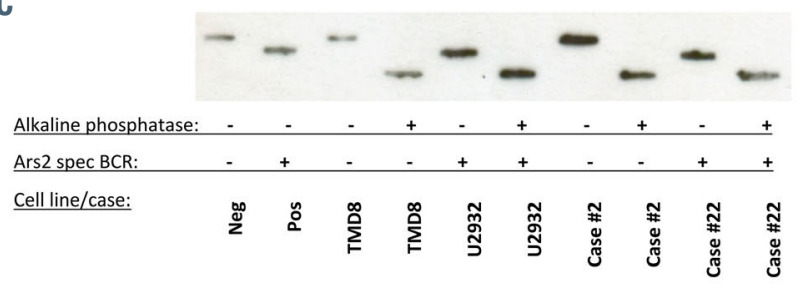

D

Cell line: $\quad$ HBL1 OCI-LY10 HBL1 OCI-LY10 HBL1 OCI-LY10 HBL1 OCI-LY10 HBL1 OCI-LY10 Mutants:
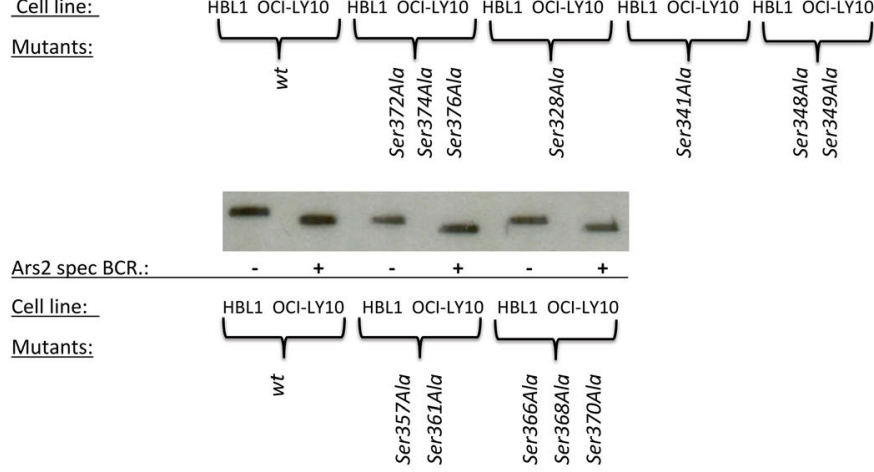

Figure 2. Ars2 is exclusively hypophosphorylated in patients with Ars2-reactivity of lymphoma B-cell receptors. (A) Western blot and isoelectric focusing (IEF) of Ars2 derived from diffuse large B-cell lymphoma (DLBCL) cell lines. Western blot of Ars2 in nine DLBCL cell lines revealed no difference in Ars2 between cell lines with and without Ars2-reactive B-cell receptors (BCR) (above). However, IEF of Ars2 in the DLBCL lines showed a less negative charge of Ars2 in OCI-Ly3, OCI-Ly10 and U2932. These three cell lines had exclusively Ars2-reactive BCR. (B) IEF of Ars2 of whole blood derived from DLBCL patients with Ars2-autoantibodies (\#22, \#27, $\# 41$, \#73). The less negatively charged Ars2 isoform was also detected In the peripheral blood of these four patients. Ars2 autoantibody titers ranged between 1:800 and 1:1600 in these four patients. (C) Alkaline phosphatase treatment led to disappearance of differences in IEF of Ars2. A stronger reduction of negative charges of Ars2 by dephosphorylation was observed in cases/cell lines without Ars2-reactive BCR. (D) Identification of the hypophosphorylated sites by site-directed mutagenesis and transfection of C-terminally FLAG-tagged Ars2 into U2932 and HBL1. In contrast to wild-type Ars2, mutations in Ser372Ala, Ser374Ala, Ser376Ala, Ser348Ala, Ser349 Ala, Ser357Ala, Ser361Ala, Ser365Ala, Ser368Ala and Ser370Ala resulted in hypophosphorylated Ars2 isoforms in both HBL1 and U2932; however the Ars2 isoform of U2932 was still less negatively charged compared to that of HBL1. Only the mutants Ser328Ala and Ser341Ala resulted in the disappearance of this difference in electric charge, identifying both Ser328 and Ser341 as the sites of hypophosphorylation. Murine anti-FLAG-antibody was used as the primary antibody. 
BCR-expressing cell lines, but had no observable effect on DLBCL lines lacking Ars2-reactive BCR (Figure 4B). No toxic effect was observed with the control toxin LRPAP1-ETA' against OCI-Ly3. Trypan blue staining after addition of $5 \mu \mathrm{g} / \mathrm{mL}$ Ars2-ETA' showed that $35 \%$, $2 \%$ and $0 \%$ of OCI-Ly3 cells were alive after $24 \mathrm{~h}, 48 \mathrm{~h}$ and $72 \mathrm{~h}$, respectively, contrasting with findings for the wild-type HBL1 cell line without BCR reactivity against Ars2 $(97 \%$ viable cells at $24 \mathrm{~h} ; 96 \%$ at $48 \mathrm{~h}$; and $97 \%$ at $72 \mathrm{~h}$ ) (Figure 4B). In accordance with this, an increase of apoptotic cells was detected after incubation with Ars2ETA' in U2932 cells expressing Ars2-reactive BCR (Figure $4 \mathrm{C}$ ), as demonstrated in the annexin $\mathrm{V} /$ propidium iodide assay.

\section{Discussion}

Beside the two relatively rare target antigens, ubiquitinated FamH83 and sumoylated JmJD4, in the present study, hypophosphorylated Ars2 was identified as a more frequent antigen of BCR from DLBCL lines and recombinant BCR from primary DLBCL cryospecimens. Ars2 is also known as serrate RNA effector molecule (SRRT). Its gene is located on chromosome $7 q 21$ and the protein is a zinc finger protein consisting of 875 amino acids with a molecular weight of around $100 \mathrm{kDa}$. Ars 2 was described as being involved in miRNA silencing by interacting with the nuclear cap binding complex, ${ }^{22}$ and as being involved in the innate immune response against RNA viruses by
A

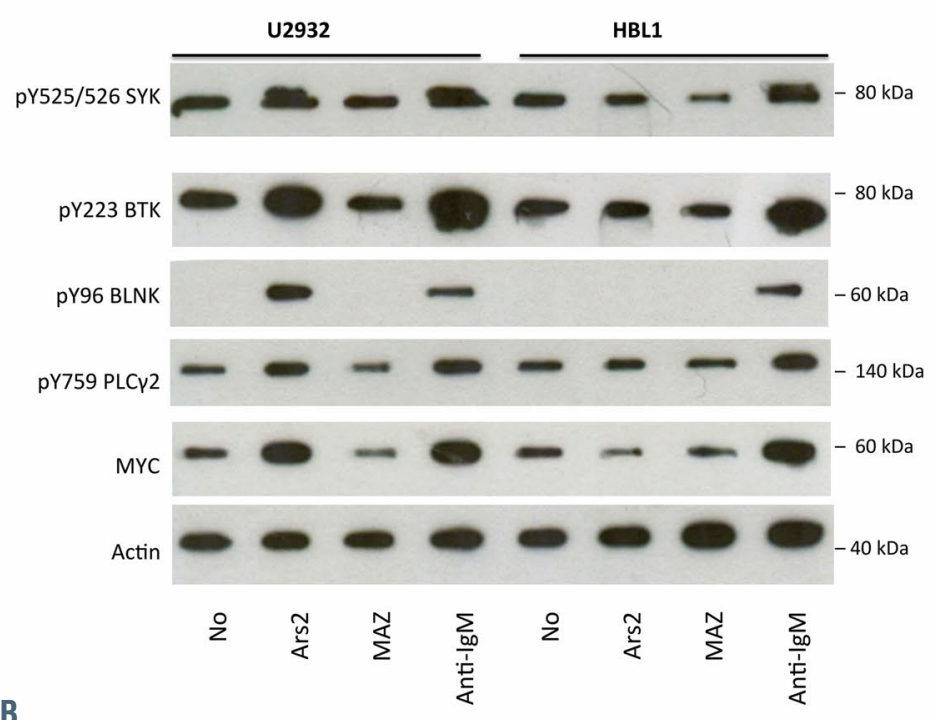

C

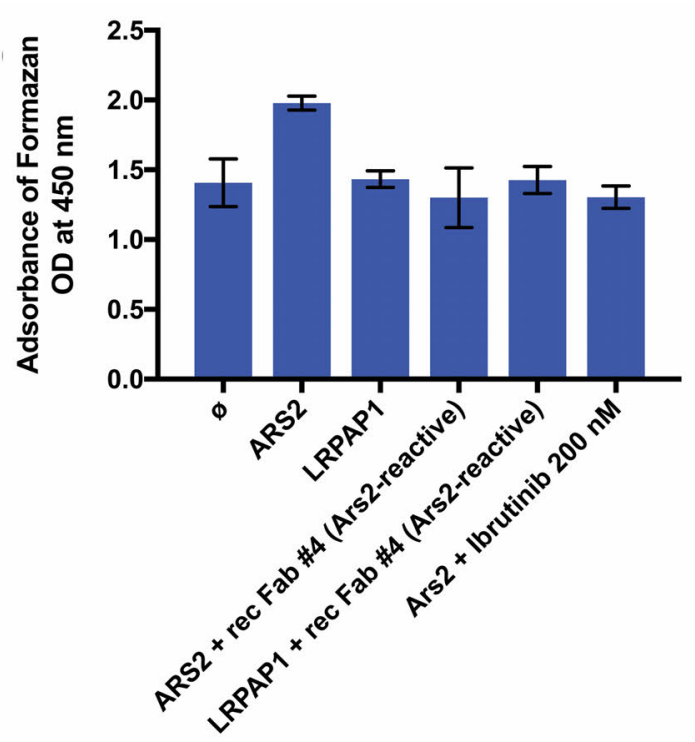

D
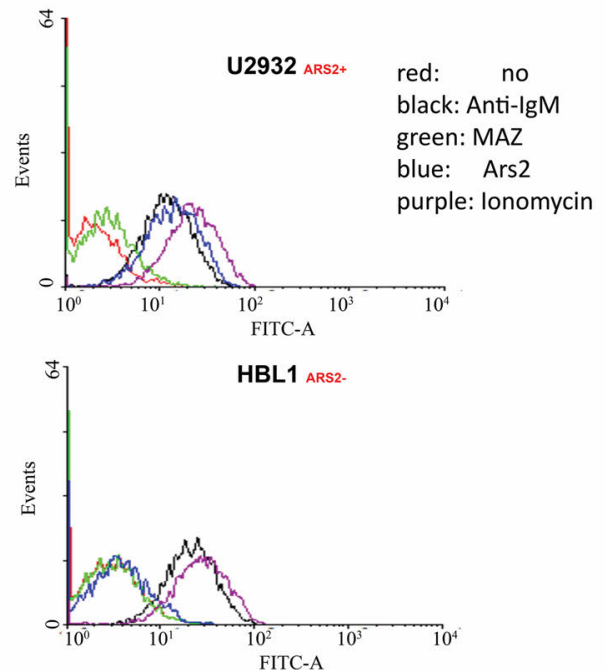

Figure 3. B-cell receptor pathway activation and induction of proliferation by Ars2. (A) B-cell receptor (BCR) pathway analysis by western blot in U2932 and HBL1 after addition of cognate/control antigens Ars2 or MAZ showed strong activation due to the addition of ARS2 to U2932 cells with upregulation of pTyr525/526 SYK, pTyr96 BLNK, pTyr759 PLCy2 and pTyr223 BTK and higher expression of MYC. In contrast, no effect of ARS2 on the BCR pathway was observed in HBL1 cells. (B) Induction of proliferation by Ars2. Addition of Ars2 to OCl-Ly3 and U2932 lines resulted in a statistically significant ( $P<0.01$ : Student $t$-test) increase of proliferation, as determined by the EZ4U assay (columns represent formazan at an optical density [OD] of $450 \mathrm{~nm}$ ), while addition of Ars2 had no effect on the TMD8 cell line. Columns and bars represent mean and standard deviation of three experiments. (C) Inhibition of Ars2-induced proliferation by neutralizing Ars2-reactive Fab. Addition of Ars2 together with Ars2-reactive (patient derived, case \#4) recombinant Fab prevented induction of growth in U2932 cells. (D) Elevation of cytoplasmatic calcium levels by addition of Ars2. Flow cytometry analysis of cytoplasmic calcium levels using Fluo-4 dye showed an increase after addition of the cognate antigen ARS2 (blue) comparable to the effect of adding anti-lgM (black) to U2932 cells, but not after the addition of a control antigen MAZ (green). Addition of Ars2 to control the diffuse large B-cell lymphoma line HBL1 did not result in elevated calcium levels. 
A

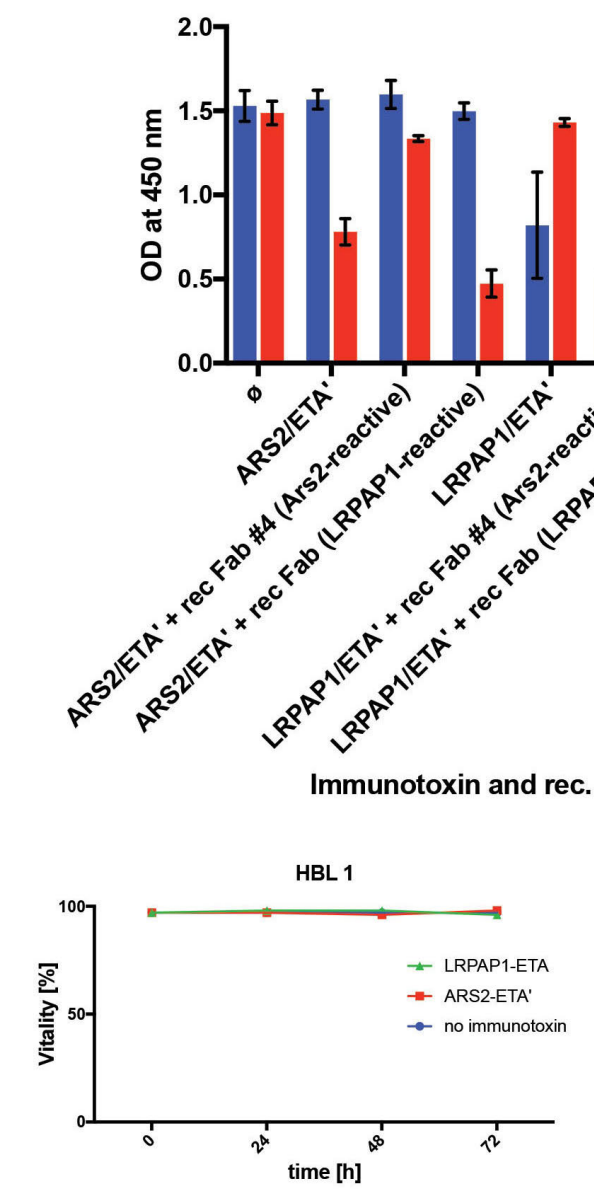

MAVER1

U2932
HBL 1

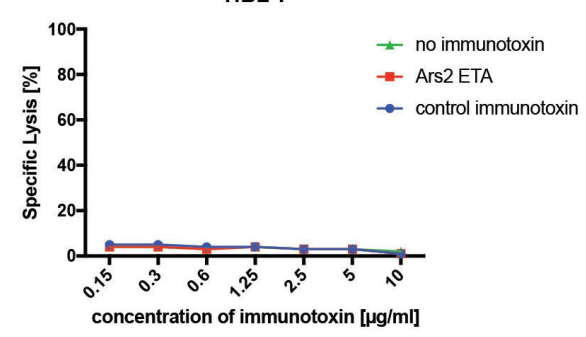

B
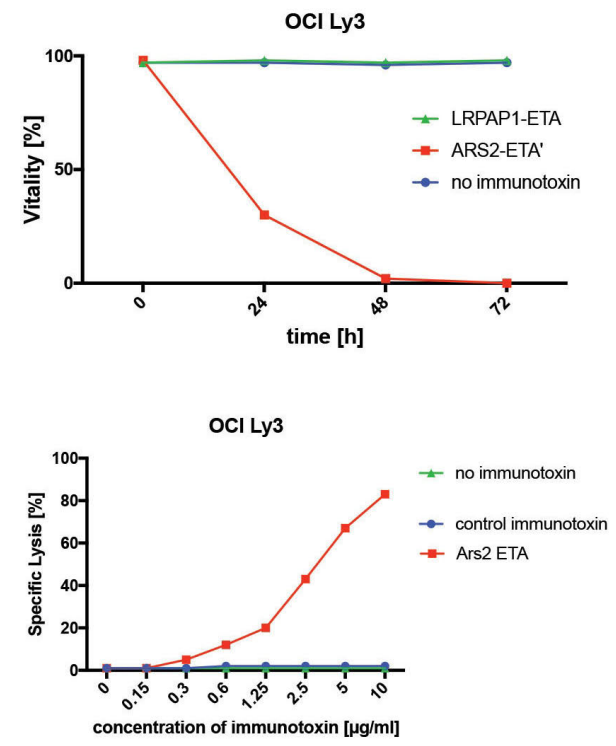

Figure 4. Targeting Ars2-reactive diffuse large B-cell lymphoma with Ars2-containing immunotoxins. (A) Growth inhibition by B-cell receptor (BCR)-antigen/immunotoxins. Growth of U2932 cells was inhibited by addition of Ars2/ETA', an immunotoxin of the epitope of the cognate antigen Ars2 fused to a truncated form of Pseudomonas aeruginosa exotoxin A. This growth inhibition could be prevented by coincubation with neutralizing Ars2reactive Fab but not by LRPAP1reactive Fab. Columns (formazan formation detected at an optical density of $450 \mathrm{~nm}$ ) represent cell proliferation (mean and standard deviation). (B) BCR-specific lysis of DLBCL cell lines by Ars2/ETA' immunotoxin. Above: cytotoxic effect after $24 \mathrm{~h}, 48 \mathrm{~h}$ and $72 \mathrm{~h}$ of incubation with $5 \mu \mathrm{g} / \mathrm{mL}$ of recombinant Ars2/ETA' or LRPAP1/ETA' immunotoxins. Cell viability of $\mathrm{HBL}$ 1 (left) and OCl-LY3 (right) cell lines determined by trypan blue staining. Below: Dose-dependent cytotoxic effect of Ars2/ETA' determined in a lactate dehydrogenase (LDH) release assay. Curves indicate percent specific lysis of the HBL1 line (left) or OCI Ly3 line (right) with and without Ars2-reactive BCR, after incubation with doses from 0 $\mu \mathrm{g} / \mathrm{mL}$ to $10 \mu \mathrm{g} / \mathrm{mL}$ Ars2/ETA', LRPAP1/ETA' or phosphatebuffered saline. (C) Induction of apoptosis by addition of Ars2/ETA' immunotoxins. Flow cytometric characterization of apoptotic U2932 or HBL1 cells $24 \mathrm{~h}$ after addition of Ars2/ETA' or MAZ/ETA' by annexin-V/propidium iodide staining. U2932 cells have Ars2reactive $\mathrm{BCR}$ resulting in a strong increase of early and late apoptotic cells after addition of Ars2/ETA'.

Ars2 reactive $\mathrm{BCR}$

모

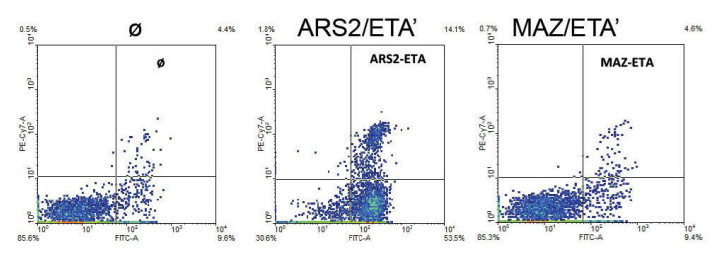

HBL1

no Ars2-reactive BCR

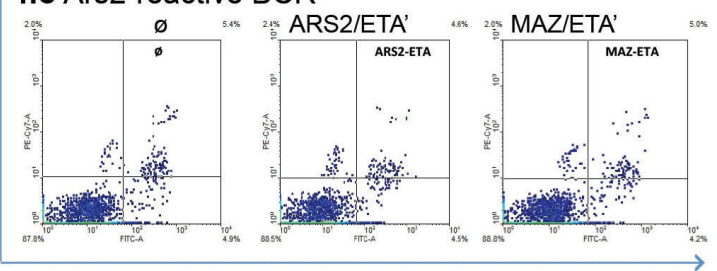

Annexin-FITC 
miRNA processing. ${ }^{23}$ Ars2 expression was shown to be linked to proliferative states ${ }^{24}$ and different roles were described in malignant disesases. ${ }^{25,26}$

All three target antigens of DLBCL-BCR identified in this study share the characteristic of being atypically post-translationally modified, which represents the most likely reason for their immunogenicity. For ubiquitinated FamH83 and sumoylated JmJD4, the lymphoma BCR were specific for the secondary modified isoforms. In contrast, Ars2-reactive BCR of DLBCL bound both the hypophosphorylated and the normally phosphorylated isoforms of Ars2. However, the hypophosphorylated isoform of Ars 2 was only observed in cell lines or cryospecimens of DLBCL with BCR reactivity for Ars2 $(P<0.0001)$. Similarly, regarding peripheral blood, the hypophosphorylated isoform of Ars2 was only observed in lysates of peripheral blood cells of patients seropositive for Ars2 autoantibodies $(P<0.0001)$. These statistically significant associations between Ars2-reactivity and the presence of hypophosphorylated Ars2 indicate that this post-translational modification is involved in the immunogenicity.

Bringing this into the context of other B-cell neoplasias, in plasma cell dyscrasia SLP2-reactive paraprotein was also not specific for the differentially phosphorylated SLP2 isoform. ${ }^{27}$ As for CD4 ${ }^{+}$T-helper cells specific for hyperphosphorylated SLP2 in plasma cell dyscrasia, ${ }^{28}$ one might speculate about a possible role of hypothetical CD4+ T-helper cells specific for hypophosphorylated Ars2 epitopes, which might stimulate Ars2-reactive B cells, which by themselves do not differentiate between normally phosphorylated and hypophosphorylated Ars2. Generally, atypical post-translational modifications represent an accepted mechanism for the breakdown of selftolerance, with numerous examples in clinical immunology, such as modified wheat gliadin in celiac disease,$^{29} \mathrm{~N}$ terminally acetylated myelin basic protein in multiple sclerosis, ${ }^{30}$ citrullinated fibrin/vimentin in rheumatoid arthritis, ${ }^{31,32}$ phosphorylated SR proteins during stressinduced apoptosis in systemic lupus erythematosus, 33,34 immunogenic pSer81 progranulin isoform and progranulin autoantibodies, ${ }^{35,36}$ phosphorylated enolase in pancreatic carcinoma, ${ }^{37-39}$ and the involvement of atypically modified BCR target antigens in lymphomagenesis including hyperphosphorylated SLP2, ATG13, and sumoylated HSP90 in plasma cell dyscrasia and hyper-Nglycosylated SAMD14/neurabin-I in primary central nervous system lymphoma. ${ }^{11,15,27,40,41}$

Of interest, Ars2 hypophosphorylation and reactivity of DLBCL BCR against Ars2 was nearly exclusively detected in DLBCL of the ABC type. All the cell lines with anti-Ars2 reactivity were of this cell of origin, and in a validation cohort of DLBCL with cell of origin characterized by GEP, hypophosphorylated Ars2 was detected in eight of 31 cases $(26 \%)$ of the ABC type of DLBCL, but in only one of 20 cases (5\%) of GCB-type DLBCL. In a combined analysis of GEP-typed cryospecimens and analyzed cell lines, the hypophosphorylated Ars2 isoform was statistically significantly associated with ABC type $(P=0.0188)$.

Considering possible functional effects of Ars2, we observed that its addition stimulated growth of DLBCL lines with Ars2-specific BCR (Figure 3), indicating that these lines still depend to some extent on BCR stimulation by their cognate antigen Ars2. Regarding the mutational background of these cell lines with Ars2-reactive BCR and expression of hypophosphorylated Ars2, OCI-Ly10 has mutated MYD88 (L265P) ${ }^{42}$ and a truncating mutation of CD79A, ${ }^{7,43}$ U2932 has a mutated NFKB-pathway by TAK1 mutation, ${ }^{44}$ but wild-type CARD11, and wild-type MYD88,75 and OCI-Ly3 has a mutated CARD11 and mutated MYD88 (L265P)., ${ }^{7,42}$ This demonstrates that, despite pathway-activating mutations, the proliferation of these cell lines might still benefit from an upstream BCR pathway stimulation by a cognate antigen.

From a therapeutic point of view, two hypothetical approaches arise from these data. Firstly, BCR antigens might be used as baits to target lymphoma cells in a specific way (i.e., targeting of a cell-bound antibody by an antigen), similarly to anti-idiotype antibodies or peptibodies, ${ }^{46}$ with the advantage of not having to be selected and synthesized individually for each patient, since all Ars2-reactive DLBCL BCR bind the same epitope. Physiologically it is the major task of a surface immunoglobulin to bind its cognate antigen and then internalize it, enabling processing and antigen presentation via MHC class II molecules. Targeting Ars2-reactive BCR of DLBCL cell lines resulted in a specific and efficacious killing. Beside this, the Ars2 epitope could be used for bispecific constructs for T- or NK-cell engaging (e.g. CD3/Ars2 or CD16/Ars2), ${ }^{47}$ or as an additional ectodomain for chimeric antigen receptor $\mathrm{T}$ cells. ${ }^{48}$ Secondly, regarding the high relative risk of carriers of atypically hypophosphorylated Ars2, investigating ways of modulating this post-translational modification might be worthwhile in the future.

\section{Disclosures}

No conflicts of interest to disclose. MP died during the preparation of the manuscript. Saarland University has applied for a relevant patent.

\section{Contributions}

LTh, SH, KDP, BK and MP designed the study. SH and $M L H$ performed microdissection of DLBCL cases and interpreted data. WK, $A W Y J K, R M B, B K, F v B, L T r, M Z, N M, D K M$ $V P$ and GH were of great help in the acquisition of DLBCL samples and clinical data. NF performed the protein array, phosphorylation and proteomic experiments. ER did site-directed point mutagenesis of Ars2. MK and ER performed sequencing studies. LTh, MK, ER and TB performed seminested IgV gene $P C R$ and $B C R$ expression cloning. LTh, MB, MK, ER and NF performed expression of Ars2-immunotoxins. LTh and MP were responsible for data analysis, interpretation of results and writing the manuscript. This article is dedicated to the memory of $M P$, who died during its preparation.

\section{Acknowledgments}

We are grateful to the entire team of the Jose-Carreras-Center for Immuno- and Gene Therapy, the DSHNHL and GLA, the Department of Internal Medicine I of Saarland University Medical School for continuous logistic and intellectual support. We also thank Hans Drexler from DSMZ for constant support.

\section{Funding}

This work was supported by a grant from Wilhelm-SanderStiftung. 


\section{References}

1. Rosenwald A, Staudt LM. Gene expression profiling of diffuse large B-cell lymphoma. Leuk Lymphoma. 2003;44(Suppl 3):S41-47.

2. Alizadeh AA, Eisen MB, Davis RE, et al. Distinct types of diffuse large B-cell lymphoma identified by gene expression profiling. Nature. 2000;403(6769):503-511.

3. Reddy A, Zhang J, Davis NS, et al. Genetic and functional drivers of diffuse large B cell lymphoma. Cell. 2017;5;171(2):481-494.

4. Lenz G, Wright G, Dave SS, et al. Stromal gene signatures in large-B-cell lymphomas. N Engl J Med. 2008;27;359(22):2313-2323.

5. Schmitz R, Wright GW, Huang DW, et al. Genetics and pathogenesis of diffuse large B-cell lymphoma. $N$ Engl J Med. 2018;12;378(15):1396-1407.

6. Chapuy B, Stewart C, Dunford AJ, et al. Molecular subtypes of diffuse large B cell lymphoma are associated with distinct pathogenic mechanisms and outcomes. Nat Med. 2018;24(5):679-690.

7. Davis RE, Ngo VN, Lenz G, et al. Chronic active B-cell-receptor signalling in diffuse large B-cell lymphoma. Nature 2010;463 (7277):88-92

8. Young RM, Wu T, Schmitz R, et al. Survival of human lymphoma cells requires B-cell receptor engagement by self-antigens. Proc Natl Acad Sci U S A. 2015;112(44):1344713454.

9. Montesinos-Rongen M, Godlewska E, Brunn A, Wiestler OD, Siebert R, Deckert $M$. Activating L265P mutations of the MYD88 gene are common in primary central nervous system lymphoma. Acta Neuropathol. 2011;122(6):791-792.

10. Montesinos-Rongen M, Schafer E, Siebert $\mathrm{R}$, Deckert M. Genes regulating the B cell receptor pathway are recurrently mutated in primary central nervous system lymphoma. Acta Neuropathol. 2012;124(6): 905-906.

11. Thurner L, Preuss K-D, Bewarder M, et al. Hyper N-glycosylated SAMD14 and neurabin-I as driver CNS autoantigens of PCNSL. Blood. 2018;132(26):2744-2753.

12. Cepok S, Zhou D, Srivastava R, et al. Identification of Epstein-Barr virus proteins as putative targets of the immune response in multiple sclerosis. J Clin Invest. 2005;115(5):1352-1360.

13. Preuss KD, Pfreundschuh M, Ahlgrimm M, et al. A frequent target of paraproteins in the sera of patients with multiple myeloma and MGUS. Int J Cancer. 2009;125(3):656661.

14. Thurner L, Müller A, Cérutti M, et al. Wegener's granuloma harbors B lymphocytes with specificities against a proinflammatory transmembrane protein and a tetraspanin. J Autoimmun. 2011;36(1):8790.

15. Preuss KD, Pfreundschuh M, Fadle N, Regitz E, Kubuschok B. Sumoylated HSP9O is a dominantly inherited plasma cell dyscrasias risk factor. J Clin Invest. 2015;125(1):316-323

16. Merbl Y, Kirschner MW. Large-scale detection of ubiquitination substrates using cell extracts and protein microarrays. Proc Natl Acad Sci U S A. 2009;106(8):2543-2548.

17. Bornkamm GW, Berens C, Kuklik-Roos C, et al. Stringent doxycycline-dependent control of gene activities using an episomal one-vector system. Nucleic Acids Res. 2005;33(16):1-11.
18. Doronina SO, Toki BE, Torgov MY, et al. Development of potent monoclonal antibody auristatin conjugates for cancer therapy. Nat Biotech. 2003;21(7):778-784.

19. Oflazoglu E, Kissler KM, Sievers EL Grewal IS, Gerber HP. Combination of the anti-CD30-auristatin-E antibody-drug conjugate (SGN-35) with chemotherapy improves antitumour activity in Hodgkin lymphoma. Br J Haematol. 2008;142(1):6973.

20. Nachreiner T, Kampmeier F, Thepen T, Fischer R, Barth S, Stocker M. Depletion of autoreactive B-lymphocytes by a recombinant myelin oligodendrocyte glycoproteinbased immunotoxin. J Neuroimmunol. 2008;195(1-2):28-35.

21. Hans CP, Weisenburger DD, Greiner TC, et al. Confirmation of the molecular classification of diffuse large B-cell lymphoma by immunohistochemistry using a tissue microarray. Blood. 2004;103(1):275-282.

22. Gruber JJ, Olejniczak SH, Yong J, LaRocca G, Dreyfuss G, Thompson CB. Ars2 promotes proper replication-dependent histone mRNA 3' end formation. Mol Cell. 2012;45(1):87-98.

23. Sabin LR, Zhou R, Gruber JJ, et al. Ars2 regulates both miRNA- and siRNA- dependent silencing and suppresses RNA virus infection in Drosophila. Cell. 2009;138(2):340351 .

24. Gruber JJ, Zatechka DS, Sabin LR, et al. Ars2 links the nuclear cap-binding complex to RNA interference and cell proliferation. Cell. 2009;138(2):328-339.

25. He Q, Huang Y, Cai L, Zhang S, Zhang C. Expression and prognostic value of Ars2 in hepatocellular carcinoma. Int J Clin Oncol. 2014;19(5):880-888.

26. Cui L, Gao C, Zhang RD, et al. Low expressions of ARS2 and CASP8AP2 predict relapse and poor prognosis in pediatric acute lymphoblastic leukemia patients treated on China CCLG-ALL 2008 protocol. Leuk Res. 2015;39(2):115-123.

27. Grass S, Preuss KD, Pfreundschuh $M$ Autosomal-dominant inheritance of hyperphosphorylated paratarg-7. Lancet Oncol. 2010;11(1):12.

28. Neumann F, Pfreundschuh M, Preuss KD, et al. CD4(+) T cells in chronic autoantigenic stimulation in MGUS, multiple myeloma and Waldenstrom's macroglobulinemia. Int J Cancer. 2015;137(5):1076-1084.

29. Arentz-Hansen H, Korner R, Molberg O, et al. The intestinal $T$ cell response to alphagliadin in adult celiac disease is focused on a single deamidated glutamine targeted by tissue transglutaminase. J Exp Med. 2000; 191(4):603-612

30. Zamvil SS, Mitchell DJ, Moore AC, Kitamura K, Steinman L, Rothbard JB. Tcell epitope of the autoantigen myelin basic protein that induces encephalomyelitis. Nature. 1986;324(6094):258-260.

31. Schellekens GA, de Jong BA, van den Hoogen FH, van de Putte LB, van Venrooij WJ. Citrulline is an essential constituent of antigenic determinants recognized by rheumatoid arthritis-specific autoantibodies. J Clin Invest. 1998;101(1):273-281.

32. van Venrooij WJ, Pruijn GJ. Citrullination: a small change for a protein with great consequences for rheumatoid arthritis. Arthritis Res. 2000;2(4):249-251.

33. Neugebauer KM, Merrill JT, Wener MH, Lahita RG, Roth MB. SR proteins are autoantigens in patients with systemic lupus erythematosus. Importance of phosphoepitopes. Arthritis Rheum. 2000;43(8) 1768-1778.

34. Utz PJ, Hottelet M, Schur PH, Anderson P. Proteins phosphorylated during stressinduced apoptosis are common targets for autoantibody production in patients with systemic lupus erythematosus. J Exp Med. 1997;185(5):843-854

35. Thurner L, Preuss KD, Fadle N, et al. Progranulin antibodies in autoimmune diseases. J Autoimmun. 2013;42:29-38.

36. Thurner L, Fadle N, Regitz E, et al. The molecular basis for development of proinflammatory autoantibodies to progranulin. J Autoimmun. 2015;61:17-28.

37. Cappello P, Tomaino B, Chiarle R, et al. An integrated humoral and cellular response is elicited in pancreatic cancer by $\alpha$-enolase, a novel pancreatic ductal adenocarcinomaassociated antigen. Int J Cancer 2009;125 (3):639-648.

38. Tomaino B, Cappello P, Capello M, et al Circulating autoantibodies to phosphorylated $\alpha$-enolase are a hallmark of pancreatic cancer. J Proteome Res. 2011;10(1):105-112

39. Zhou W, Capello M, Fredolini C, et al Mass spectrometry analysis of the posttranslational modifications of alpha-enolase from pancreatic ductal adenocarcinoma cells. J Proteome Res. 2010;9(6):29292936.

40. Grass S, Preuss KD, Wikowicz A, et al. Hyperphosphorylated paratarg-7: a new molecularly defined risk factor for monoclonal gammopathy of undetermined significance of the IgM type and Waldenstrom macroglobulinemia. Blood. 2011;117(10) 2918-2923.

41. Preuss KD, Pfreundschuh M, Fadle N, et al. Hyperphosphorylation of autoantigenic targets of paraproteins is due to inactivation of PP2A. Blood. 2011;118(12):33403346.

42. Ngo VN, Young RM, Schmitz R, et al Oncogenically active MYD88 mutations in human lymphoma. Nature. 2011;470 (7332):115-121.

43. Gordon MS, Kanegai CM, Doerr JR, Wall $R$. Somatic hypermutation of the $B$ cell receptor genes $\mathrm{B} 29(\mathrm{Ig} \beta, \mathrm{CD} 79 \mathrm{~b})$ and $\mathrm{mb} 1$ (Ig $\alpha$, CD79a). Proc Natl Acad Sci U S A. 2003;100(7):4126-4131.

44. Compagno M, Lim WK, Grunn A, et al. Mutations of multiple genes cause deregulation of NF-kappaB in diffuse large B-cel lymphoma. Nature. 2009;459(7247):717 721.

45. Mondello P, Brea EJ, De Stanchina E, et al Panobinostat acts synergistically with ibrutinib in diffuse large B cell lymphoma cells with MyD88 L265 mutations. JCI Insight. 2017;2(6):e90196.

46. Torchia J, Weiskopf K, Levy R. Targeting lymphoma with precision using semisynthetic anti-idiotype peptibodies. Proc Nat Acad Sci U S A. 2016;113(19):5376-5381.

47. Bewarder M, Preuss KD, Fadle N, Regitz E, Thurner L, Pfreundschuh M. CD3/Bars: a novel bispecific format for the treatment of B-cell lymphomas. Blood. 2016;128(22) 3516.

48. Kochenderfer JN, Dudley ME, Kassim SH, et al. Chemotherapy-refractory diffuse large B-cell lymphoma and indolent B-cell malignancies can be effectively treated with autologous $T$ cells expressing an antiCD19 chimeric antigen receptor. J Clin Oncol. 2015;33(6):540-549. 